\title{
Cost-Utility Analysis of Sacubitril-Valsartan Compared with Enalapril Treatment in Patients with Acute Decompensated Heart Failure in Thailand
}

\author{
Rungroj Krittayaphong ${ }^{1} \cdot$ Unchalee Permsuwan ${ }^{2}$ (I)
}

Accepted: 31 August 2021 / Published online: 17 September 2021

(c) The Author(s), under exclusive licence to Springer Nature Switzerland AG 2021

\begin{abstract}
Background Sacubitril-valsartan is effective in reducing the N-terminal pro-B-type natriuretic peptide level of hospitalized patients with acute decompensated heart failure, with a high acquisition cost compared with enalapril treatment.

Objective This study aimed to determine the cost utility of sacubitril-valsartan compared with enalapril for acute decompensated heart failure treatment.

Methods A Markov model was constructed to project the total costs, life-years, quality-adjusted life-years (QALYs) of early initiation, and a 2-month delay of sacubitril-valsartan treatment and enalapril treatment in hospitalized patients with acute decompensated heart failure over a lifetime horizon from a Thai healthcare system perspective. Clinical inputs were mainly derived from the PIONEER-HF and PARADIGM-HF trials, together with Thai epidemiological data. Cost data were based on the Thai population. All costs and outcomes were discounted at 3\% annually. A series of sensitivity analyses were performed. Results Compared with enalapril, sacubitril-valsartan incurred a higher total cost per year (THB 42,994 [US\$1367.48] vs THB 19,787 [US\$629.37]), and it gained more QALYs (4.969 vs 4.755). The incremental cost-effectiveness ratio was THB 108,508/QALY (US\$3451.26/QALY). Early initiation of sacubitril-valsartan treatment was more cost effective than delayed treatment. Sensitivity analyses revealed that at a level of willingness to pay of THB 160,000/QALY (US\$5089/ QALY), sacubitril-valsartan was a cost-effective strategy of about $60 \%$.

Conclusions Sacubitril-valsartan is cost effective in patients with acute decompensated heart failure. However, the results are highly dependent on the long-term cardiovascular mortality, and they are applicable only to Thailand or countries with a similarly structured healthcare system. Long-term registries should be pursued to decrease the uncertainty around longterm mortality.
\end{abstract}

\section{Introduction}

Heart failure (HF) is a leading cardiovascular (CV) problem worldwide [1]. Its prevalence is increasing, and the mortality rate is high [2]. It was estimated that the 1-year and 5-year mortality rates after HF hospitalization were approximately $30 \%$ and $60 \%$, respectively [3], which were similar to data reported for the Asian population [4]. The mortality rate was

Unchalee Permsuwan

unchalee.permsuwan@cmu.ac.th

1 Division of Cardiology, Department of Medicine, Faculty of Medicine Siriraj Hospital, Mahidol University, Bangkok, Thailand

2 Department of Pharmaceutical Care, Faculty of Pharmacy, Chiang Mai University, 239 Huaykaew Road, Suthep, Chiang Mai 50200, Thailand higher in patients with recent HF hospitalization than in those with chronic HF [5, 6]. The readmission rate after HF hospitalization was high [6]. Approximately $20 \%$ of patients were readmitted because of HF within 1 month of discharge following HF hospitalization [7]. The readmission rate was higher in low-income or low-income to middle-income countries [7]. There were many reasons for the high readmission rate [8]. A major reason was suboptimal HF-medication optimization [9]. According to the practice guideline for the management of patients with $\mathrm{HF}$ with reduced ejection fraction, patients are required to have many guideline-directed medical therapies, such as renin-angiotensin system blockers, beta-blockers, and mineralocorticoid receptor antagonists [10, 11]. Moreover, physicians need to up-titrate the dose of each medication until the maximally tolerated dose is reached. Treatment inertia is another important factor [12]. Recently, both sacubitril valsartan, which acts as an angiotensin receptor-neprilysin inhibitor 


\section{Key Points}

Sacubitril-valsartan has been used in stable heart failure with reduced ejection fraction.

The PIONEER-HF study shows the benefit of sacubitrilvalsartan in hospitalized patients with acute decompensated heart failure.

Sacubitril-valsartan is a cost-effective treatment for patients with acute decompensated heart failure, compared with enalapril, in the Thai context.

(ARNi), and sodium-glucose co-transporter 2 inhibitors have been shown to have an impact on the clinical outcomes of patients with HF with reduced ejection fraction [13, 14]. Practice guidelines have recommended that ARNi be used to replace renin-angiotensin system blockers [10]. Moreover, ARNis can be used in patients who are renin-angiotensin system blocker naive [13]. To avoid delayed initiation of beneficial drugs such as ARNis, the trial entitled PIONEERHF (Comparison of Sacubitril-Valsartan versus Enalapril on Effect on NT-proBNP in Patients Stabilized From an Acute Heart Failure Episode) showed that ARNi can be safely initiated during hospitalization for acute decompensated heart failure (ADHF) [15]. The primary outcome was the change in the N-terminal pro-B-type natriuretic peptide levels at weeks 4 and 8 . The results demonstrated that the ARNi group had a greater reduction in the $\mathrm{N}$-terminal pro-B-type natriuretic peptide level than the placebo group. The safety outcomes of the two groups were similar.

The development of new drugs is typically expensive. Moreover, population aging, the resultant increase in chronic diseases, and the COVID-19 pandemic have imposed severe economic pressures on the sustainability of healthcare systems. Health economic evaluations, such as cost-utility analyses, have been used as a tool in many countries, including Thailand. These studies generate economic evidence that enable decision makers to rationally allocate available healthcare resources by comparing the costs and outcomes of treatment strategies [16]. In Thailand, economic evidence of costly innovative health technology, such as new drugs, is required to support its inclusion in the National List of Essential Medicine. The evidence also informs coverage decisions related to benefits packages in the country's Universal Coverage Scheme [17]. A new treatment is deemed cost effective when the incremental cost-effectiveness ratio is less than THB 160,000 (US\$5089.06) per 1 qualityadjusted life-year (QALY) gained [18]. To support healthcare-provider decisions related to HF treatment, this study compared the cost utilities of sacubitril-valsartan treatment and enalapril treatment in patients with acute HF.

\section{Methods}

\subsection{Cohort Population}

The modeled population had equivalent characteristics to patients enrolled in the PIONEER-HF clinical trial [15]. The PIONEER-HF study also assessed the efficacy and safety of the initiation of sacubitril-valsartan therapy, compared with enalapril therapy, in patients stabilized from an acute $\mathrm{HF}$ episode. Its trial period was 8 weeks. In brief, our patients were at least 18 years of age; had a left ventricular ejection fraction of $\leq 40 \%$; had a $\mathrm{N}$-terminal pro-B-type natriuretic peptide level of $\geq 1600 \mathrm{pg} / \mathrm{mL}$, or a B-type natriuretic peptide level of $\geq 400 \mathrm{pg} / \mathrm{mL}$; had acute decompensated HF; and were hospitalized. The average age of the Thai patients with HF was 60 years, which was used as the starting age in this study.

\subsection{Intervention and Comparator}

The Thai Health Technology Assessment Guideline recommends using the current practice as the comparator when conducting a cost-effectiveness study [19]. Enalapril is a commonly used medicine for HF treatment in Thailand. Therefore, a target dose of $10 \mathrm{mg}$ of enalapril, twice daily, was used as the comparator in the current investigation. Based on the 8-week trial period of the PIONEER-HF trial, we adopted two interventions for the present work: the immediate administration of sacubitril-valsartan, and a 2-month delay in its administration. More specifically, the intervention in the first patient group started with a target of $97 \mathrm{mg}$ of sacubitril and $103 \mathrm{mg}$ of valsartan, given twice daily, during the first month of hospitalization. The medication continued to be administered until the lifetime horizon. As to the second patient group, their treatment commenced with $10 \mathrm{mg}$ of enalapril, which was given twice daily during months 1 and 2 only. From month 3 onwards, sacubitrilvalsartan was used instead of enalapril.

\subsection{Model Structure}

We used a Markov model that was based on our previous sacubitril-valsartan cost-effectiveness study and the work of Gaziano et al. [20, 21]. The model comprised five health states: hospitalized HF; non-hospitalized HF: month 1; nonhospitalized HF: month 2; non-hospitalized HF: month 3 and over; and death (CV and non-CV; Fig. 1). To capture the long-term costs and outcomes, a lifetime horizon with a 1-month cycle length was used for analyses.

The cohort population was hospitalized patients with HF. At the start of the cycle, the cohort population could remain hospitalized, could improve and be discharged from hospital 
(non-hospitalized HF: month 1), or become worse and die from $\mathrm{CV}$ causes. For the next cycle, the non-hospitalized patients with HF could remain non-hospitalized (non-hospitalized: HF month 2), be readmitted to hospital, or die from $\mathrm{CV}$ causes. If patients were still non-hospitalized in the subsequent cycle, they were moved to non-hospitalized HF: month 3. Patients with HF who were non-hospitalized for at least 3 consecutive months died either from $\mathrm{CV}$ or non- $\mathrm{CV}$ causes. Patients in any non-hospitalized health state were readmitted to the hospital.

\subsection{Input Parameters}

All input parameters are detailed in Table 1.

\subsubsection{Transitional Probabilities}

For the first two cycles, the transitional probabilities were based on the 8-week trial period of the PIONEER-HF trial [15]. If the patients with HF were non-hospitalized for 2 months, we assumed that their HF symptoms would become stable and have a profile similar to the population from the trial entitled PARADIGM-HF (Prospective Comparison of ARNi with ACEI to Determine Impact on Global Mortality and Morbidity in Heart Failure) [22]. This assumption was based on the work of Gaziano et al. [21]. Therefore, the transitional probabilities for cycle 3 and onwards were obtained from PARADIGM-HF, which was conducted on a stable HF with reduced ejection fraction population.

2.4.1.1 Cardiovascular Death During the PIONEER-HF study period, there were ten $(2.27 \%) \mathrm{CV}$ deaths out of 440

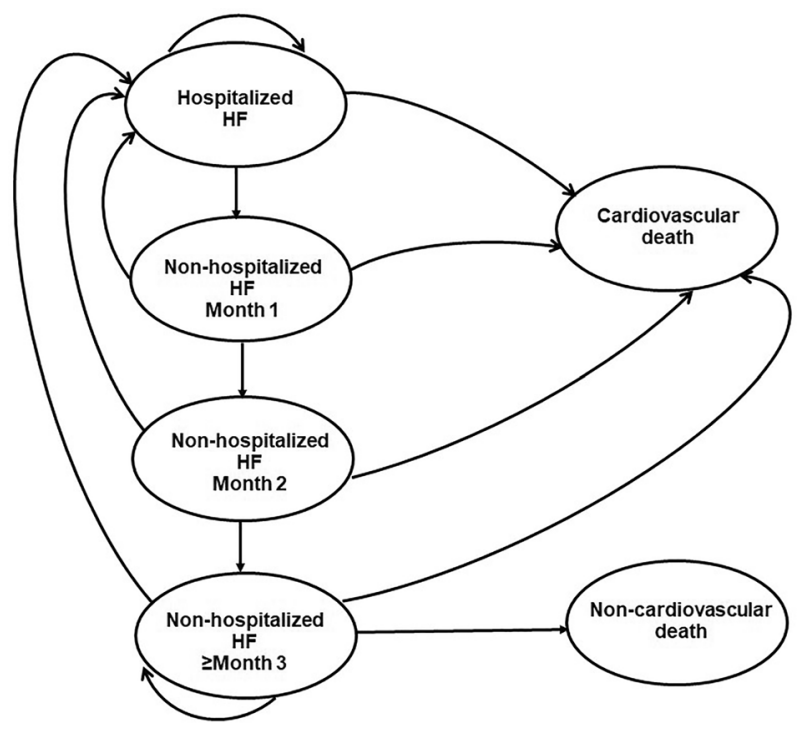

Fig. 1 Markov model for patients with acute decompensated heart failure (HF) patients receiving sacubitril-valsartan, and $15(3.40 \%) \mathrm{CV}$ deaths out of 441 patients receiving enalapril. [15] These probabilities were converted to a 1-month rate using the formula, $r=-[\ln (1-p)] / t$, where $p$ is probability, $r$ is rate, and $t$ is duration. The rates were subsequently converted back to 1-month probabilities. This gave 0.011 for the sacubitril-valsartan group and 0.017 for the enalapril group.

For the third month and onwards, the risk of $\mathrm{CV}$ death was obtained from the PARADIGM-HF trial [22]. It reported $558(13.33 \%) \mathrm{CV}$ deaths out of 4187 patients in the sacubitril-valsartan group, and 693 (16.45\%) CV deaths out of 4212 patients in the enalapril group. The median followup time was 27 months. These risks were subsequently converted to 1-month probabilities: sacubitril-valsartan, 0.005 ; and enalapril, 0.007 .

2.4.1.2 Non-Cardiovascular Death Age-specific mortality rates for the Thai general population, with $\mathrm{CV}$ deaths removed, were obtained from the Ministry of Public Health, Thailand [23]. Patients with HF had a higher risk of death than patients without HF. Based on the work by Corrao et al. [24], deaths occurred more frequently among hospitalized HF cohort members than non-HF cohort members. Their cumulative mortalities were $24 \%$ and $2.8 \%$, respectively, yielding a relative risk of 8.571 . This risk value was used to adjust the Thai general age-specific mortality rates for patients with HF.

2.4.1.3 Hospitalization The risk of rehospitalization for the first two cycles was based on data from the PIONEER trial [15]. In that investigation, 35 patients out of 440 patients in a sacubitril-valsartan group, and 61 patients out of 441 patients in an enalapril group, were readmitted, resulting in 1-month probabilities of 0.041 and 0.072 , respectively. For the third month and onwards, the rate of readmission in the enalapril group was obtained from the Thai HF National database, which reported a $34 \%$ readmission rate [25]. This rate was converted to a 1 -month probability of 0.288 . The PARADIGM-HF trial reported an odds ratio (95\% confidence interval) for readmission of $0.74(0.56-0.97)$ [26]. The odds ratio was converted to relative risk using the following formula: relative risk $=$ odds ratio/[ $(1-$ prevalence of the outcome in unexposed group) + (prevalence of the outcome in unexposed group $\times$ odds ratio)]. The relative risk was then multiplied by the 1-month probability of readmission for the enalapril group. This gave the 1-month probability of readmission for the sacubitril-valsartan group: 0.231.

\subsubsection{Costs}

Given that the present work adopted the perspective of the healthcare system, it only included direct medical costs (the costs of hospitalization and drug acquisition). Based on the 
Table 1 Input parameters

\begin{tabular}{|c|c|c|c|c|}
\hline Parameters & Value & Range & Distribution & References \\
\hline \multicolumn{5}{|l|}{ Risk of CV death (1-2 months) } \\
\hline Enalapril $^{\mathrm{a}}$ & 0.017 & $0.015-0.019$ & Beta & \multirow[t]{2}{*}{ Velazquez et al. [15] } \\
\hline Sacubitril-valsartan $^{\mathrm{b}}$ & 0.011 & $0.010-0.013$ & Beta & \\
\hline \multicolumn{5}{|l|}{ Risk of CV death ( $\geq 3$ months) } \\
\hline Enalapril $^{\mathrm{c}}$ & 0.007 & $0.006-0.007$ & Beta & \multirow[t]{2}{*}{ McMurray et al. [21] } \\
\hline Sacubitril-valsartan $^{\mathrm{d}}$ & 0.005 & $0.005-0.006$ & Beta & \\
\hline \multicolumn{4}{|l|}{ Risk of non-CV death by age $\geq 60$ years } & \multirow[t]{5}{*}{ Ministry of Public Health [22] } \\
\hline 60 & 0.008 & & & \\
\hline 65 & 0.012 & & & \\
\hline$\geq 70$ & 0.035 & & & \\
\hline 100 & 0.069 & & & \\
\hline \multicolumn{5}{|l|}{ Risk of rehospitalization (1-2 months) } \\
\hline Enalapril $^{\mathrm{e}}$ & 0.072 & $0.065-0.079$ & Beta & \multirow[t]{2}{*}{ Velazquez et al. [15] } \\
\hline Sacubitril-valsartan ${ }^{\mathrm{f}}$ & 0.041 & $0.037-0.045$ & Beta & \\
\hline \multicolumn{5}{|l|}{ Risk of rehospitalization ( $\geq 3$ months) } \\
\hline Enalapril ${ }^{\mathrm{g}}$ & 0.288 & $0.259-0.317$ & Beta & Thailand database [24] \\
\hline Sacubitril-valsartan ${ }^{\mathrm{h}}$ & 0.231 & $0.208-0.254$ & Beta & Calculation \\
\hline Relative risk of heart failure & 8.571 & $7.714-9.429$ & Log-normal & Corrao et al. [23] \\
\hline \multicolumn{5}{|l|}{ Costs (THB/US\$) } \\
\hline \multicolumn{5}{|l|}{ Medications (1 month) } \\
\hline Enalapril & $24.90(0.79)$ & $19.92-29.8(0.63-0.95)$ & Gamma & \multirow[t]{2}{*}{ DMSIC [26] } \\
\hline Sacubitril-valsartan & $4413.60(140.38)$ & $3530.88-5296.32(112.31-168.46)$ & Gamma & \\
\hline Hospitalization & $\begin{array}{r}11,576.79 \\
(368.22)\end{array}$ & $9261.43-13,892.14(294.57-441.86)$ & Gamma & Krittayaphong et al. [28] \\
\hline \multicolumn{5}{|l|}{ Utility } \\
\hline \multicolumn{5}{|l|}{ Heart failure } \\
\hline Enalapril & 0.829 & $0.746-0.912$ & Beta & \multirow[t]{2}{*}{ Gazziano et al. [32] } \\
\hline Sacubitril-valsartan & 0.838 & $0.754-0.922$ & Beta & \\
\hline Utility decrement from hospitalization & 0.100 & $0.090-0.110$ & Gamma & King et al. [31] \\
\hline
\end{tabular}

$C V$ cardiovascular, DMSIC Drug and Medical Supply Information Center, THB Thai Baht, US\$ US dollars

${ }^{\mathrm{a}} 1$-Month rate $=-[\ln (1-2 / 441)] / 2=0.017$. 1-Month probability $=1-\exp (-0.017)=0.017$

${ }^{\mathrm{b}} 1$-Month rate $=-[\ln (1-10 / 440)] / 2=0.011$. 1-Month probability $=1-\exp (-0.011)=0.011$

${ }^{\mathrm{c}} 1$-Month rate $=-[\ln (1-693 / 4212)] / 27=0.007$. 1-Month probability $=1-\exp (-0.007)=0.007$

${ }^{\mathrm{d}} 1$-Month rate $=-[\ln (1-558 / 4187)] / 27=0.005$. 1-Month probability $=1-\exp (-0.005)=0.005$

${ }^{\mathrm{e}} 1-$ Month rate $=-[\ln (1-61 / 441)] / 2=0.074$. 1-Month probability $=1-\exp (-0.074)=0.072$

${ }^{\mathrm{f}} 1$-Month rate $=-[\ln (1-35 / 440)] / 2=0.041$. 1-Month probability $=1-\exp (-0.041)=0.041$

${ }^{\mathrm{g}}$ Risk of all-cause readmission at 30 days of enalapril $=1-\exp (-0.34)=0.288$

${ }^{\mathrm{h}}$ Relative risk of readmission $=0.74 /[(1-0.288)+(0.288 \times 0.74)]=0.80 .1$-Month probability $=0.80 \times 0.288=0.231$

PARADIGM-HF trial, patients receiving sacubitril-valsartan had a significant number of symptomatic hypotension events than those receiving enalapril ( $14 \%$ vs $9.2 \%, p<0.001)$. However, patients receiving sacubitril-valsartan experienced a lower number of elevated serum creatinine $(\geq 2.5 \mathrm{mg} / \mathrm{dL})$, serum potassium, and cough than those receiving enalapril (3.3\% vs $4.5 \%, p=0.007 ; 4.3 \%$ vs $5.6 \%, p=0.007 ; 11.3 \%$ vs $14.3 \%, p<0.001$, respectively) [22]. These significant adverse events incur low treatment costs. As a result, we decided to exclude the cost of adverse events. Furthermore, we assumed that all other direct costs incurred with the two treatments would not differ and would be cancelled out in the analysis.

The drug costs per day were calculated from the daily dose and unit cost. The dose of enalapril was $10 \mathrm{mg}$ twice daily. The unit costs of enalapril and sacubitril-valsartan were obtained from the Drug and Medical Supply Information Center, Ministry of Public Health [27]. In cases where a product was supplied by several pharmaceutical companies, the median drug price from the median list 
of all pharmaceutical companies was used, in accordance with the Thai Health Technology Assessment Guideline [28]. The cost of enalapril was THB $0.83 /$ day (US $\$ 0.03 /$ day), or THB $24.90 /$ month (US\$0.79/day). The cost of sacubitril-valsartan was THB 147.12/day (US\$4.68/day), or THB 4413.60/month (US\$140.38/month). The cost of HF hospitalization was based on our previous study [29], which obtained its data from the Central Office for Health Care Information, National Health Security Office. This center gathers data on the healthcare expenditure on the $85 \%$ of the Thai population who are covered by the Civil Servant Medical Benefit Scheme and the Universal Coverage Scheme. The average cost \pm standard deviation was THB 11,576.79 $\pm 15,162.15$ (US $\$ 368.22 \pm 482.26$ ). All costs were inflated to the year of 2020 using the consumer price index for the medical-care category [30]. The costs were converted to US dollars at the rate of THB 31.44 per US Dollar [31].

\subsubsection{Utility}

The utility data were adopted from other published HF studies. Being hospitalized gave patients with HF a disutility of 0.1 [32]. Patients receiving sacubitril-valsartan had a slightly higher utility than those receiving enalapril (0.838 vs 0.829$)$ [33].

\subsection{Cost-Utility Analysis}

\subsubsection{Base-Case Analysis}

The outcomes of interest in this study were total costs, lifeyears, QALYs, and incremental cost-effectiveness ratio (ICER). The total costs, life-years, and QALYs of sacubitril-valsartan and enalapril were estimated and discounted at an annual rate of 3\%, as per the Thai Health Technology Assessment Guideline [34]. The ICER was calculated as the difference in total cost divided by the difference in QALYs or life-years between sacubitril-valsartan and enalapril. The Thai acceptable ceiling ratio of a cost-effective alternative was THB 160,000/QALY, or US\$5089/QALY [18]. If the estimated ICER of sacubitril-valsartan compared with enalapril fell below that ratio, sacubitril-valsartan treatment would be considered a cost-effective strategy. In addition, sacubitril-valsartan treatment had a lower number of CV deaths than enalapril treatment per 1000 patients with acute HF (471 vs 585 CV deaths).

\subsubsection{Sensitivity Analyses}

A one-way sensitivity analysis was performed to explore the impact of the individual parameter uncertainties on the results. Transition probabilities, utilities, and relative risk were varied within the range of $\pm 10 \%$, whereas all costs were varied within the range of $\pm 20 \%$. All plausible ranges are summarized in Table 1 . The discount rate varied from 0 to $6 \%$, following the recommendation of the Thai Health Technology Assessment Guideline [34]. The results are displayed as a Tornado diagram.

In addition, a probabilistic sensitivity analysis was conducted whereby individual sets of parameter values were drawn from appropriate statistical distributions (Table 1), with results generated for 1000 simulation runs. The results of the analysis were output as a scatterplot and a cost-effectiveness acceptability curve. These graphically represented the probability of sacubitril-valsartan being cost effective, compared with enalapril, for different defined willingnessto-pay thresholds.

\section{Results}

\subsection{Base-Case Analysis}

The results of the cost-utility analysis demonstrated that sacubitril-valsartan treatment incurred a higher total cost per year (THB 42,994 [US\$1367.48] vs THB 19,787 [US\$629.37]) and gained more life-years (6.096 vs 5.941) and QALYs (4.969 vs 4.755) than enalapril treatment. This yielded an incremental cost of THB 23,206 (US\$738.12), an incremental life-year of 0.155 , and an incremental QALY of 0.214. As a result, the ICER was THB 149,893/ life-year (US\$4767.60/life-year) or THB 108,508/QALY (US\$3451.26/QALY) (Table 2).

When patients with acute HF received 2 months of enalapril before switching to sacubitril-valsartan treatment, the incremental cost incurred was THB 22,084 (US\$702.41) with 0.088 life-year gained or 0.157 QALY gained, compared with those who received enalapril treatment. This yielded an ICER of THB 250, 960/life-year (US 7982.19/ life-year) or THB 140,276/life-year (US\$4461.71/QALY).

\subsection{Sensitivity Analyses}

The results of the one-way sensitivity analysis using a tornado diagram showed that variation in the risk of $\mathrm{CV}$ death with enalapril treatment from 3 months onwards had the strongest impact on the ICER estimate. This was followed by the risk of CV death with sacubitril-valsartan treatment from 3 months onwards. The drug acquisition cost of sacubitril valsartan had slight effects on the ICER (Fig. 2). The scatter plot on the cost-effectiveness plane (Fig. 3) demonstrated that all 1000 simulations fell on the upper quadrant. This signified that sacubitril-valsartan treatment was more costly than enalapril treatment. Of those 1000 simulations, $74 \%$ fell on the upper-right quadrant, indicating that sacubitril-valsartan treatment gained more QALYs than enalapril treatment 
Table 2 Results of the base-case analysis

\begin{tabular}{|c|c|c|c|c|c|c|c|c|}
\hline \multirow[t]{2}{*}{ Treatment } & \multirow[t]{2}{*}{ Total cost (THB/US\$) } & \multirow[t]{2}{*}{ Total LYs } & \multirow[t]{2}{*}{ Total QALYs } & \multicolumn{3}{|l|}{ Incremental } & \multirow{2}{*}{$\begin{array}{l}\text { ICER (THB/ } \\
\text { LY)/(US\$/ } \\
\text { LY) }\end{array}$} & \multirow{2}{*}{$\begin{array}{l}\text { ICER (THB/ } \\
\text { QALY)/ (US\$/ } \\
\text { QALY) }\end{array}$} \\
\hline & & & & Cost (THB/US\$) & LY & QALY & & \\
\hline Enalapril $^{\mathrm{a}}$ & $19,787(629.37)$ & 5.941 & 4.755 & & & & & \\
\hline $\begin{array}{l}\text { Enalapril for } 2 \mathrm{mo} \text {, } \\
\text { then sacubitril- } \\
\text { valsartan }\end{array}$ & $\begin{array}{l}41,871 \\
(1331.78)\end{array}$ & 6.029 & 4.913 & $22,084(702.41)$ & 0.088 & 0.157 & $\begin{array}{l}250,960 \\
(7982.19)\end{array}$ & $\begin{array}{l}140,276 \\
(4461.71)\end{array}$ \\
\hline Sacubitril-valsartan & 42,994 (1367.48) & 6.096 & 4.969 & $\begin{array}{l}23,206 \\
(738.12)\end{array}$ & 0.155 & 0.214 & $\begin{array}{l}149,893 \\
(4767.60)\end{array}$ & $\begin{array}{l}108,508 \\
(3451.26)\end{array}$ \\
\hline
\end{tabular}

ICER incremental cost-effectiveness ratio, $L Y$ life-year, mo months, $Q A L Y$ quality-adjusted life-year, $T H B$ Thai Baht, US\$ US dollars

${ }^{a}$ Enalapril was a comparator

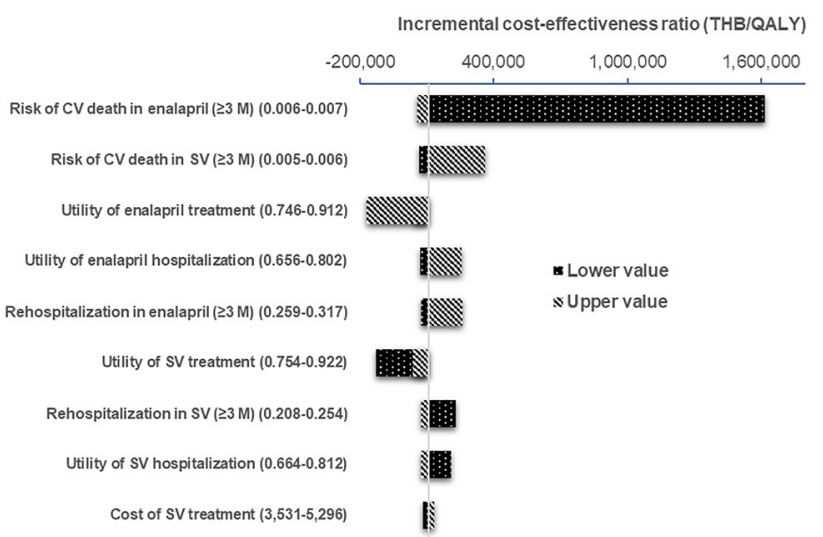

Fig. 2 Tornado diagram presenting the results of the one-way sensitivity analysis (sacubitril-valsartan vs enalapril). $C V$ cardiovascular, $M$ months, $Q A L Y$ quality-adjusted life-year, $S V$ sacubitril-valsartan, THB Thai Baht

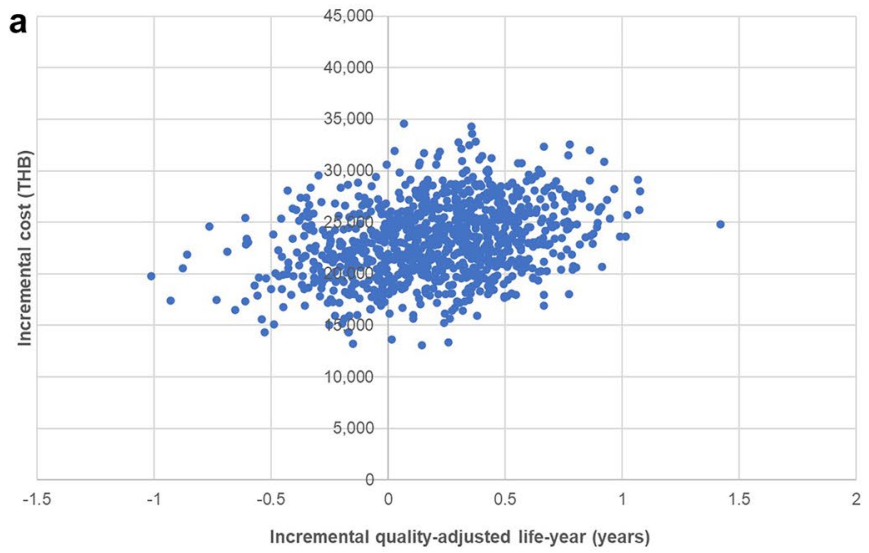

(Fig. 3A). The results of the probabilistic sensitivity analysis (Fig. 4) indicated that approximately $60 \%$ of the 1000 simulations fell below the THB 160,000/QALY local threshold when sacubitril-valsartan was compared with enalapril for acute HF treatment.

\section{Discussion}

The findings of this study indicated that treatment of acute HF with sacubitril-valsartan combination therapy compared with enalapril had an ICER of THB 108,508/ QALY (US\$3451.26/QALY). This is considered a costeffective strategy, given the local threshold in Thailand. Early initiation of sacubitril-valsartan treatment would be more cost effective than delayed treatment, with ICERs of THB 108,508/QALY (US\$3451.26/QALY) and

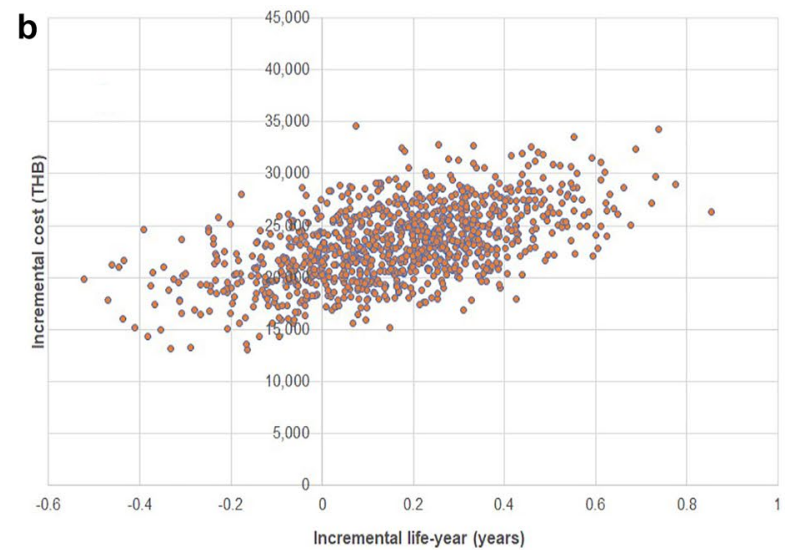

Fig. 3 Scatter plot of 1000 simulations (sacubitril-valsartan vs enalapril) on the cost-effectiveness plane. a Incremental cost and incremental quality-adjusted life-years. b Incremental cost and incremental life-years. THB Thai Baht 


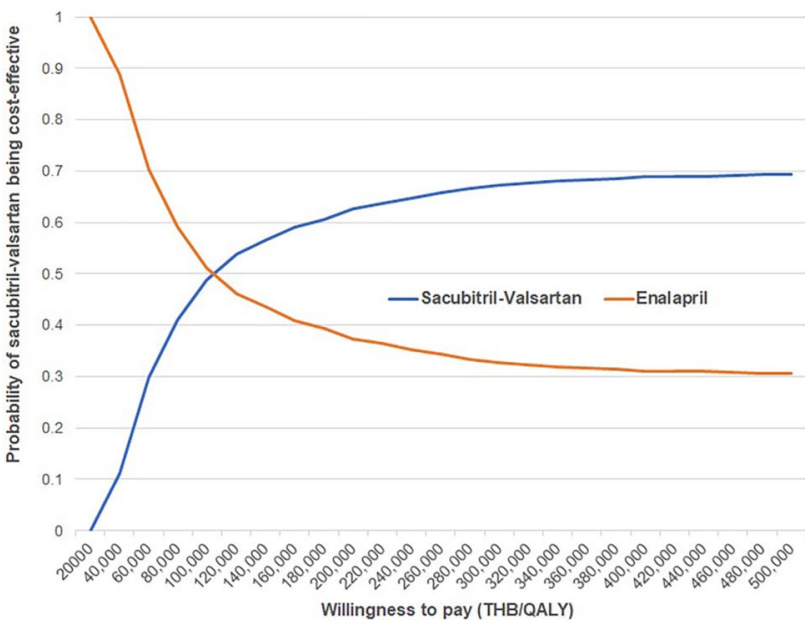

Fig. 4 Cost-effectiveness acceptability curve showing the results of the probabilistic sensitivity analysis (sacubitril-valsartan vs enalapril). $Q A L Y$ quality-adjusted life-year, $T H B$ Thai Baht

THB 140,276/QALY (US\$4461.71/QALY), respectively. The probabilistic sensitivity analysis findings from 1000 iterations showed that about $60 \%$ of the iterations had an ICER falling below the local threshold.

Based on the one-way sensitivity analysis, the estimated ICER was sensitive to the risk of CV death in enalapril treatment from month 3 onwards. The lower range generated the greater ICER, while the upper range yielded the lower ICER. This implies that the small risk of CV death in the enalapril group results in longer life-years and QALYs. The incremental benefit gained from receiving sacubitril-valsartan treatment would be less, leading to a high ICER (THB 1.63 million; Fig. 2). Further research related to $\mathrm{CV}$ deaths of patients with HF receiving enalapril treatment should be considered.

Since the publication of the PARADIGM HF study, there have been many cost-effectiveness analyses (CEAs) to determine the cost effectiveness of using ARNis in patients with chronic HF. Those investigations have been conducted in a number of countries, such as the USA, the UK, Australia, Germany, and Korea [35]. We also reported our CEA data on ARNi for chronic HF. That work was based on the event rate in the Thai population and the magnitude of benefit provided by the PARADIGM HF study. We found that the cost of ARNis would need to be reduced by $2 \%$ to achieve an ICER below the threshold of THB 160,000 per QALY [20]. Cost-effectiveness analyses may differ from country to country. In our case, the CEA results depended on the input parameters from local data, i.e., the annual CV mortality rate from hospitalization in Thailand, the risk of all-cause readmission at 30 days for enalapril, and the risk of nonCV mortality. The level of willingness to pay per QALY is another important factor contributing to the differences in the CEA results among countries. The willingness to pay for Thailand is THB 160,000 per QALY; this is generally smaller than the corresponding values from other countries, especially high-income countries [18].

Following the release of the PIONEER HF trial results, several studies have published CEAs of the use of ARNis in hospitalized patients with ADHF [21, 36]. Gaziano et al. reported CEA data relating to hospitalized patients with ADHF. Their work found that ARNi initiation during hospitalization saved US $\$ 452$ per year, compared with continuation of enalapril. In a comparison involving the initiation of ARNis 2 months after hospitalization, the cost saving was US $\$ 811$ per year. In addition, the ICER was US\$21,532 per QALY, compared with continued enalapril treatment over a lifetime [21]. However, interpretation of those CEA results requires much caution. The results of the treatment effect, which was an input of the Markov model, were obtained from a carefully planned, randomized clinical trial in a specific setting of patients who fitted the inclusion and exclusion criteria. The results may therefore not be generalized to realworld clinical practice. The input parameters of the CEA model also required many assumptions based on theoretical postulations. Therefore, sensitivity analyses are required to assess CEA models in different settings [37]. Another CEA of ARNis in hospitalized patients with ADHF, conducted in Australia and based on a Markov model, determined that ARNis were not cost effective. A price reduction of more than $25 \%$ was needed to confer cost effectiveness [36].

The results of our study confirmed earlier CEAs of ARNis in a hospital setting for ADHF [21]. Our study is an example of CEA data of ARNis in hospitalized patients with ADHF in the Asian population. Initiation of ARNis during hospitalization with ADHF was cost effective, relative to continuing with enalapril. This result firmly supports the use of ARNis early in the course of HF rather than waiting a few months until HF becomes more chronic. Heart failure has a malignant course, high mortality, and a high readmission rate. The administration of a beneficial drug early in the course of the disease may change the natural history of the disease and improve prognosis. Each hospital admission of ADHF involves additional myocardial damage and increased progression of the disease.

Previous data demonstrated that patients with a recent hospital admission because of ADHF had a higher rate of readmission and death than those with chronic HF [5, 6]. Therefore, providing a treatment that has proven benefits, particularly during ADHF, would be more advantageous to patients than delaying initiation until the chronic phase. A clinical trial is required to demonstrate that a medication that is beneficial for chronic HF can be initiated during hospital admission. This is because during admission, patients may be unstable, and a medication may not be safe to initiate. Angiotensin receptor-neprilysin inhibitors have already 
demonstrated their efficacy and safety both in chronic HF and ADHF settings. A CEA is another step towards determining whether ARNis are value-based treatments [16].

The decision model for the reimbursement of a new drug may need to take into account not only the findings of a CEA but also the payment policies of each country [18, 37]. In some situations, it may be appropriate to have a co-pay system in which patients meet part of the medication costs. Nevertheless, some patients may be reluctant to use the drug because of the costs that would be involved [38, 39]. Therefore, cost transparency and shared decision making are needed before initiating a new drug [37].

Our study has some limitations. First, we drew upon data from the PIONEER-HF study for the risk of $\mathrm{CV}$ death and rehospitalization, and for the effects on reductions and outcomes. These data may differ among regions. However, a sub-analysis of the PARADIGM-HF study demonstrated that the benefits of sacubitril-valsartan usage were evident across regions [40]. Second, we used utility data from a published study. We addressed this limitation by conducting the sensitivity analysis. The results revealed that the utility data had no significant influence on the estimated ICER. Third, we tried to incorporate local national data, such as the risk of non-CV death and cost of hospitalization, into our model to reflect the application of the model to Thailand. There may be a limited generalizability of our findings to other nations that have different healthcare systems.

\section{Conclusions}

Sacubitril-valsartan was shown to be cost effective in patients with acute HF. However, the results are highly dependent on long-term CV mortality, and they are applicable only to Thailand or countries with similarly structured healthcare systems. While model-based analyses can help to facilitate an appreciation of cost and effectiveness drivers, long-term registries should be pursued to decrease the uncertainties around long-term mortality.

\section{Declarations}

Funding There was no funding support for this study.

Conflicts of Interest The authors report that there are no relationships that could be construed as a conflict of interest.

Ethics approval This study did not involve participants as information was gathered from previously published research; thus, no ethical approval was required.

Consent to participate Not applicable.

Consent for Publication Not applicable.
Availability of data and material Not applicable.

Code availability Not applicable.

Authors' contributions RK: research design; data acquisition and interpretation; and manuscript drafting and critical review. UP: data acquisition; model development and analysis; results interpretation; and manuscript drafting and critical review.

\section{References}

1. Virani SS, Alonso A, Aparicio HJ, Benjamin EJ, Bittencourt MS, Callaway CW, et al. Heart disease and stroke statistics-2021 update: a report from the American Heart Association. Circulation. 2021;143(8):e254-743.

2. Savarese G, Lund LH. Global public health burden of heart failure. Card Fail Rev. 2017;3(1):7-11.

3. Ponikowski P, Anker SD, AlHabib KF, Cowie MR, Force TL, Hu $S$, et al. Heart failure: preventing disease and death worldwide. ESC Heart Fail. 2014;1(1):4-25.

4. Krittayaphong R, Karaketklang K, Yindeengam A, Janwanishstaporn S. Heart failure mortality compared between elderly and non-elderly Thai patients. J Geriatr Cardiol. 2018;15(12):718-24.

5. Tavazzi L, Senni M, Metra M, Gorini M, Cacciatore G, Chinaglia A, et al. Multicenter prospective observational study on acute and chronic heart failure: one-year follow-up results of IN-HF (Italian Network on Heart Failure) outcome registry. Circ Heart Fail. 2013;6(3):473-81.

6. Armstrong PW, Pieske B, Anstrom KJ, Ezekowitz J, Hernandez $\mathrm{AF}$, Butler $\mathrm{J}$, et al. Vericiguat in patients with heart failure and reduced ejection fraction. N Engl J Med. 2020;382(20):1883-93.

7. Reddy YNV, Borlaug BA. Readmissions in heart failure: it's more than just the medicine. Mayo Clin Proc. 2019;94(10):1919-21.

8. Naik N, Narula J. Heart failure in low-income and middle-income countries: failing REPORT card grades. Lancet Glob Health. 2020;8(3):e318

9. Tromp J, Bamadhaj S, Cleland JGF, Angermann CE, Dahlstrom U, Ouwerkerk W, et al. Post-discharge prognosis of patients admitted to hospital for heart failure by world region, and national level of income and income disparity (REPORT-HF): a cohort study. Lancet Glob Health. 2020;8(3):e411-22.

10. Ponikowski P, Voors AA, Anker SD, Bueno H, Cleland JG, Coats AJ, et al. ESC Guidelines for the diagnosis and treatment of acute and chronic heart failure: the Task Force for the Diagnosis and Treatment of Acute and Chronic Heart Failure of the European Society of Cardiology (ESC). Developed with the special contribution of the Heart Failure Association (HFA) of the ESC. Eur Heart J. 2016;37(27):2129-200.

11. Yancy CW, Jessup M, Bozkurt B, Butler J, Casey DE Jr, Colvin MM, et al. 2017 ACC/AHA/HFSA focused update of the 2013 ACCF/AHA guideline for the management of heart failure: a report of the American College of Cardiology/American Heart Association Task Force on clinical practice guidelines and the Heart Failure Society of America. J Am Coll Cardiol. 2017;70(6):776-803.

12. Verhestraeten C, Heggermont WA, Maris M. Clinical inertia in the treatment of heart failure: a major issue to tackle. Heart Fail Rev. 2020. https://doi.org/10.1007/s10741-020-09979-z

13. Writing C, Maddox TM, Januzzi JL Jr, Allen LA, Breathett K, Butler J, et al. 2021 update to the 2017 ACC Expert Consensus Decision Pathway for Optimization of Heart Failure Treatment: answers to 10 pivotal issues about heart failure with reduced ejection fraction: a Report of the American College of 
Cardiology Solution Set Oversight Committee. J Am Coll Cardiol. 2021;77(6):772-810.

14. McMurray JJV, Packer M. How should we sequence the treatments for heart failure and a reduced ejection fraction? A redefinition of evidence-based medicine. Circulation. 2021;143(9):875-7.

15. Velazquez EJ, Morrow DA, DeVore AD, Duffy CI, Ambrosy AP, McCague K, et al. Angiotensin-neprilysin inhibition in acute decompensated heart failure. N Engl J Med. 2019;380(6):539-48.

16. Di Tanna GL, Chen S, Bychenkova A, Wirtz HS, Burrows KL, Globe G. Economic evaluations of pharmacological treatments in heart failure patients: a methodological review with a focus on key model drivers. Pharmacoecon Open. 2020;4(3):397-401.

17. Leelahavarong P, Doungthipsirikul S, Kumluang S, Poonchai A, Kittiratchakool N, Chinnacom D, et al. Health technology assessment in Thailand: institutionalization and contribution to healthcare decision making: review of literature. Int J Technol Assess Health Care. 2019;35(6):467-73.

18. Thavorncharoensap M, Teerawattananon Y, Natanant S, Kulpeng $\mathrm{W}$, Yothasamut J, Werayingyong P. Estimating the willingness to pay for a quality-adjusted life year in Thailand: does the context of health gain matter? Clinicoecon Outcomes Res. 2013;5:29-36.

19. Tanvejsilp P, Ngorsuraches S. Defining the scope of health technology assessment and types of health economic evaluation. J Med Assoc Thai. 2014;97(Suppl. 5):S10-6.

20. Krittayaphong R, Permsuwan U. Cost-effectiveness analysis of sacubitril-valsartan compared with enalapril in patients with heart failure with reduced ejection fraction in Thailand. Am J Cardiovasc Drugs. 2018;18(5):405-13.

21. Gaziano TA, Fonarow GC, Velazquez EJ, Morrow DA, Braunwald E, Solomon SD. Cost-effectiveness of sacubitril-valsartan in hospitalized patients who have heart failure with reduced ejection fraction. JAMA Cardiol. 2020;5(11):1236-44.

22. McMurray JJ, Packer M, Desai AS, Gong J, Lefkowitz MP, Rizkala AR, et al. Angiotensin-neprilysin inhibition versus enalapril in heart failure. N Engl J Med. 2014;371(11):993-1004.

23. Strategy and Planning Division, Ministry of Public Health. Public health statistics A.D. 2019. Ministry of Public Health; Nonthaburi, 2020.

24. Corrao G, Ghirardi A, Ibrahim B, Merlino L, Maggioni AP. Burden of new hospitalization for heart failure: a population-based investigation from Italy. Eur J Heart Fail. 2014;16(7):729-36.

25. Janwanishstaporn $\mathrm{S}$. National trend, in-hospital and long term outcomes in the hospitalized heart failure patients in Thailand. Thai Heart J. 2015;28:128.

26. Desai AS, Claggett BL, Packer M, Zile MR, Rouleau JL, Swedberg K, et al. Influence of sacubitril/valsartan (LCZ696) on 30-day readmission after heart failure hospitalization. J Am Coll Cardiol. 2016;68(3):241-8.

27. Drug and Medical Supply Information Center, Ministry of Public Health. 2021. http://dmsic.moph.go.th. Accessed 28 Feb 2021.

28. Riewpaiboon A. Measurement of costs for health economic evaluation. J Med Assoc Thai. 2014;97(Suppl. 5):S17-26.

29. Krittayaphong R, Permsuwan U. Cost-utility analysis of add-on dapagliflozin treatment in heart failure with reduced ejection fraction. Int J Cardiol. 2021;1(322):183-90.

30. Bureau of Trade and Economics Indices, Ministry of Commerce. CPI. 2021. http://www.price.moc.go.th/price/cpi/index_new_all. asp. Accessed 28 Feb 2021.

31. Bank of Thailand. Foreign exchange rates 2020. https://www. bot.or.th/english/statistics/financialmarkets/exchangerate/_layou ts/application/exchangerate/ExchangeRate.aspx. Accessed 9 Apr 2021.

32. King JB, Shah RU, Bress AP, Nelson RE, Bellows BK. Costeffectiveness of sacubitril-valsartan combination therapy compared with enalapril for the treatment of heart failure with reduced ejection fraction. JACC Heart Fail. 2016;4(5):392-402.

33. Gaziano TA, Fonarow GC, Claggett B, Chan WW, DeschaseauxVoinet C, Turner SJ, et al. Cost-effectiveness analysis of sacubitril/valsartan vs enalapril in patients with heart failure and reduced ejection fraction. JAMA Cardiol. 2016;1(6):666-72.

34. Permsuwan U, Guntawongwan K, Buddhawongsa P. Handling time in economic evaluation studies. J Med Assoc Thai. 2014;97(Suppl. 5):S50-8.

35. Liu XQ, He LS, Huang JQ, Xiong LJ, Xia C, Lao HY. Cost-effectiveness analyses of sacubitril-valsartan for heart failure. Heart Fail Rev. 2021;26:1119-1130.

36. Perera K, Ademi Z, Liew D, Zomer E. Sacubitril-valsartan versus enalapril for acute decompensated heart failure: a cost-effectiveness analysis. Eur J Prev Cardiol. 2019;4:2047487319878953.

37. Yancy CW, Hernandez AF, Bonow RO. The use of sacubitril/ valsartan for hospitalized heart failure: why do we care about cost and value? JAMA Cardiol. 2020;5(11):1244.

38. Smith GH, Shore S, Allen LA, Markham DW, Mitchell AR, Moore M, et al. Discussing out-of-pocket costs with patients: shared decision making for sacubitril-valsartan in heart failure. J Am Heart Assoc. 2019;8(1):e010635.

39. Dickert NW, Mitchell AR, Venechuk GE, Matlock DD, Moore MA, Morris AA, et al. Show me the money: patients' perspectives on a decision aid for sacubitril/valsartan addressing out-of-pocket cost. Circ Cardiovasc Qual Outcomes. 2020;13(12):e007070.

40. Kristensen SL, Martinez F, Jhund PS, Arango JL, Belohlavek J, Boytsov S, et al. Geographic variations in the PARADIGM-HF heart failure trial. Eur Heart J. 2016;37(41):3167-74. 